\title{
Tension-sensitive Plk1 phosphorylation on BubR1 regulates the stability of kinetochore-microtubule interactions
}

\author{
Sabine Elowe, ${ }^{3}$ Stefan Hümmer, Andreas Uldschmid, ${ }^{1}$ Xiuling $\mathrm{Li}^{2}{ }^{2}$ and Erich A. $\mathrm{Nigg}^{4}$ \\ Department of Cell Biology, Max Planck Institute of Biochemistry, D-82152 Martinsried, Germany
}

\begin{abstract}
Mitotic phosphorylation of the spindle checkpoint component BubR1 is highly conserved throughout evolution. Here, we demonstrate that BubR1 is phosphorylated on the Cdk1 site T620, which triggers the recruitment of Plk1 and phosphorylation of BubR1 by Plk1 both in vitro and in vivo. Phosphorylation does not appear to be required for spindle checkpoint function but instead is important for the stability of kinetochore-microtubule (KT-MT) interactions, timely mitotic progression, and chromosome alignment onto the metaphase plate. By quantitative mass spectrometry, we identify S676 as a Plk1-specific phosphorylation site on BubR1. Furthermore, using a phospho-specific antibody, we show that this site is phosphorylated during prometaphase, but dephosphorylated at metaphase upon establishment of tension between sister chromatids. These findings describe the first in vivo verified phosphorylation site for human BubR1, identify Plk1 as the kinase responsible for causing the characteristic mitotic BubR1 upshift, and attribute a KT-specific function to the hyperphosphorylated form of BubR1 in the stabilization of KT-MT interactions.
\end{abstract}

[Keywords: BubR1; tension; phosphorylation; spindle assembly checkpoint]

Supplemental material is available at http://www.genesdev.org.

Received April 4, 2007; revised version accepted July 19, 2007.

The BubR1 protein was first identified as an essential and evolutionarily conserved component of the spindle assembly checkpoint (SAC). During mitosis, this checkpoint inhibits the anaphase-promoting complex/cyclosome (APC/C), a large E3 ubiquitin ligase, so that chromosome segregation is triggered only after bipolar attachment to the spindle microtubules (MTs) has been achieved (Musacchio and Hardwick 2002; Bharadwaj and Yu 2004; Taylor et al. 2004). SAC dysfunction and the consequential premature APC/C activation can result in aneuploidy, a hallmark of human solid tumors (Kops et al. 2005). Throughout prophase and prometaphase, SAC components including BubR1 are enriched at kinetochores (KTs), complex proteinaceous structures assembled on centromeric DNA, where a "wait anaphase" signal is thought to be generated. KTs represent the major point of contact between mitotic spindle MTs and chromosomes (Maiato et al. 2004; Chan et al. 2005). Several proteins and multiprotein complexes, including BubR1 (Lampson and Kapoor 2005; Matsumura et al. 2007) and the Hec1/Ndc80 complex (McCleland et al.

Present addresses: ${ }^{1}$ Viramed Biotech AG, Behringerstrasse 11, D-82152, Planegg, Germany; ${ }^{2}$ Group 1803, Dalian Institute of Chemical Physics, Chinese Academy of Science, 457 Zhongshan Rd. Dalian 116023, China. Corresponding authors.

${ }^{3}$ E-MAIL elowe@biochem.mpg.de; FAX 49-89-8578-3102.

${ }^{4}$ E-MAIL nigg@biochem.mpg.de; FAX 49-89-8578-3102.

Article is online at http://www.genesdev.org/cgi/doi/10.1101/gad.436007.
2004; DeLuca et al. 2006; Dong et al. 2007), have been implicated in the establishment and maintenance of the highly dynamic KT-MT interactions that are required for chromosome movement during mitosis (Chan et al. 2005; Kline-Smith et al. 2005; Rieder 2005). In addition, $\mathrm{KT}$-associated proteins contribute to maintain SAC activity until the last chromosome has undergone bipolar attachment. Although the exact nature of SAC signaling is not well understood, biochemical and genetic evidence indicate that the source of this signal is at the centromere/KT, where tension generated upon amphitelic attachment is sensed (Pinsky and Biggins 2005; Baumann et al. 2007). How such a mechanical property is transduced into a biochemical signaling cascade remains unclear, but there is strong evidence that phosphorylation plays a key role (Gorbsky 1995).

Along with APC/C-mediated proteolyis, M-phase progression is controlled through phosphorylation by mitotic kinases. In addition to the key regulator cyclindependent kinase 1 (Cdk1), Polo-like kinase 1 (Plk1) and Aurora family kinases are crucial for accurate mitotic progression. The importance of these kinases is underscored by their dynamic localization patterns, which reflect diverse functions at different stages of mitosis (Nigg 2001; Barr et al. 2004; Vagnarelli and Earnshaw 2004; van Vugt and Medema 2005). In particular, recent reports have emphasized the role of both Aurora B and Plk1 at the centromere and $\mathrm{KT}$, respectively, where they have 
been implicated in tension-responsive signal transduction, KT-MT attachment, and chromosome congression (Ditchfield et al. 2003; Sumara et al. 2004; Ahonen et al. 2005; Wong and Fang 2005; Cimini et al. 2006; Hanisch et al. 2006; Nishino et al. 2006; King et al. 2007; Lenart et al. 2007).

One of the most conspicuous and evolutionarily conserved mitotic phosphorylation events occurs on BubR1, resulting in a characteristic electrophoretic upshift. In this report, we have investigated the functions of BubR1 hyperphosphorylation during mitotic progression. We demonstrate that BubR1 is a substrate of Plk1, both in vitro and in vivo, and that Plk1 is responsible for the characteristic mitotic upshift. Moreover, we show that BubR1 association with Plk1 is enhanced by Cdk1 phosphorylation, and that cells expressing a BubR1 mutant unable to associate with Plk1 exhibit reduced KT-fiber stability, slow mitotic progression, and impaired chromosome congression. In contrast, we found no evidence that mitotic hyperphosphorylation of BubR1 is required for its association with components of the mitotic checkpoint complex (MCC) or the APC/C. Identification of an in vivo Plk1 phosphorylation site on BubR1 and probing this site with phospho-specific antibodies demonstrate a clear correlation between BubR1 phosphorylation on Ser 676 (S676) and lack of tension. Based on these observations, we propose that phosphorylation of BubR1 by Plk1 is required for the stabilization of KT-MT interactions during mammalian mitosis, and that phosphorylation of BubR1 on S676 during chromosome congression provides a read-out for the establishment of tension between sister KTs.

\section{Results}

\section{BubR1 is a mitotic binding partner and target of Plk1}

To identify kinases that might be responsible for mitotic phosphorylation of human BubR1, we expressed candidate upstream kinases in Sf9 insect cells and assayed their ability to phosphorylate full-length, bacterially expressed hBubR1 (Fig. 1A). Under these in vitro conditions, BubR1 was strongly phosphorylated by Cdk1 and Plk1, but not by Bub1, BubR1, Mps1, Aurora A, or Aurora $\mathrm{B}$ (Fig. 1A, lanes 7,8 ), even though all kinases displayed activity against appropriate substrates (data not shown). Next, we asked whether Plk1 and BubR1 can interact in vivo and performed reciprocal coimmunoprecipitation experiments, using lysates of asynchronously growing, nocodazole- or Taxol-arrested HeLa cells. As shown by Western blotting, Plk1 could readily be detected in BubR1 immunoprecipitates, and similarly, BubR1 was present in Plk1 immunoprecipitates (Fig. 1B). The mitotic kinase Mps1 was not present in either BubR1 or Plk1 immunoprecipitates, emphasizing the specificity of the BubR1-Plk1 interaction. Furthermore, the BubR1-Plk1 interaction was M-phase specific, as no significant binding was observed in immunoprecipitates from asynchronous HeLa cell lysates.
To determine whether Plk1 also phosphorylates BubR1 in vivo, we analyzed the upshift of BubR1 in nocodazole-arrested cells, in cells depleted of Plk1 by small interfering RNA (siRNA), and in cells depleted of the kinesin-related motor Eg5 (which arrest at a comparable prometaphase state) (Fig. 1C). As expected, nocodazole treatment of HeLa cells led to the appearance of the characteristic slowly migrating BubR1 band. This upshifted band could also be seen after depletion of Eg5, but not after depletion of Plk1 (Fig. 1C), indicating that it is dependent on Plk1, in excellent agreement with recent reports (Lenart et al. 2007; Matsumura et al. 2007). Thus, our data indicate that BubR1 and Plk1 are mitotic binding partners and that BubR1 is an in vitro and in vivo substrate of Plk1.

The Plk1-BubR1 interaction is mediated by PBD binding to pT620 of BubR1 and enhanced by Cdk1 phosphorylation

The phosphorylation of Plk1 targets often requires prior docking of the kinase through its C-terminal Polo-Box domain (PBD) to phosphothreonine or phosphoserine motifs on the target protein (Elia et al. 2003). To determine if the Plk1 PBD is alone sufficient for association with BubR1, we performed interaction studies with immobilized GST-tagged PBD (GST-PBD ${ }^{\mathrm{wt}}$ ) and a PBD mutant in which two residues critical for ligand binding (His 538 and Lys 540) were changed to alanine (GST$\mathrm{PBD}^{\mathrm{AA}}$. GST-PBD ${ }^{\mathrm{WT}}$, but not $\mathrm{GST}-\mathrm{PBD}^{\mathrm{AA}}$, readily pulled down BubR1 from mitotic cell lysates, demonstrating that the PBD is sufficient to mediate the interaction of Plk1 with BubR1 (Fig. 1D). Since both Cdk1 and Plk1 can act as priming kinases for generating PBD docking sites (Elia et al. 2003; Neef et al. 2003, 2007) and both kinases phosphorylate BubR1 efficiently (Fig. 1A), we used a Far Western assay to test the ability of PBD to bind BubR1 with or without prior phosphorylation by Cdk1 or Plk1 (Fig. 1E). As a positive control for PBD binding, we included the kinesin-like motor Mklp2, on which Plk1-mediated phosphorylation was previously shown to create a docking site (Neef et al. 2003). Indeed, GST-PBD strongly bound to Mklp2 that had been phosphorylated by Plk1, but not to BSA (Fig. 1E). In contrast, BubR1 phosphorylated by Plk1 alone, or nonphosphorylated BubR1, showed little PBD binding. Significantly stronger PBD binding was seen after prephosphorylation of BubR1 by Cdk1, or Cdk1 followed by Plk1, indicating that Cdk1 can generate a PBD docking site on BubR1. In addition, BubR1 immunoprecipitated from mitotic cells arrested by nocodazole or Taxol treatment bound efficiently to PBD (Fig. 1E, right lanes), in agreement with the pull-down experiments (Fig. 1D).

The BubR1-related kinase Bub1 was recently shown to interact with Plk1 through a conserved STP motif (Qi et al. 2006). A corresponding motif, centered around T620, is also present in BubR1. To test the possibility that this motif represents the Plk1 docking site on BubR1, we 

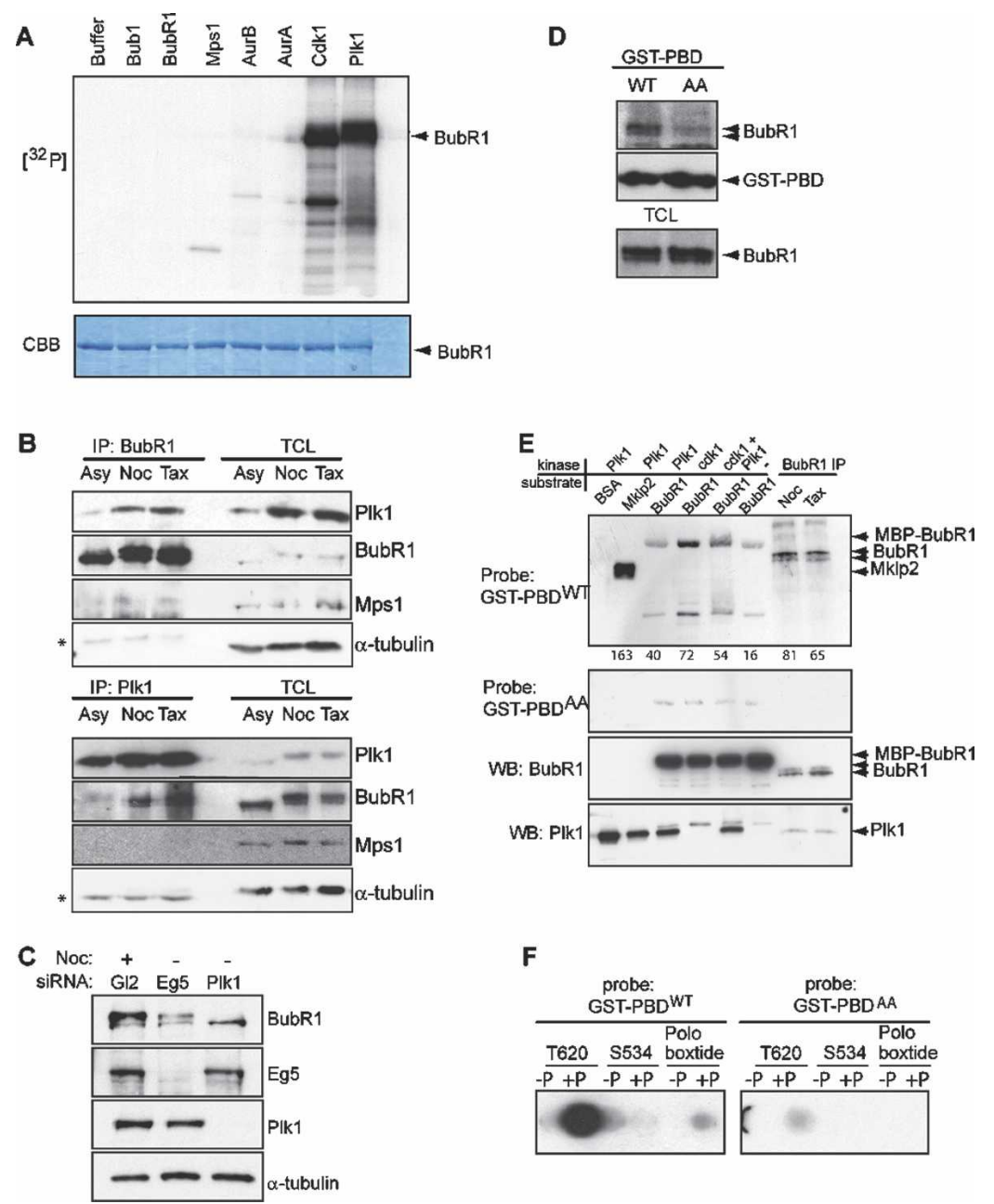

Figure 1. Hyperphosphorylation of BubR1 is regulated in vitro and in vivo by Cdk1 and Plk1. (A) In vitro kinase assays with indicated kinases or buffer alone. Protein phosphorylation is visualized by autoradiography $\left(\left[{ }^{32} \mathrm{P}\right]\right.$, top panel) and equal protein loading by Coomassie Blue staining (CBB, bottom panel). (B) Immunoprecipitation of endogenous BubR1 (top panels) or endogenous Plk1 (bottom panels) from asynchronously growing (Asy) and nocodazole-arrested (Noc) or Taxol-arrested (Tax) HeLa cells. Immunoprecipitates and corresponding total cell lysates (TCL) were probed by Western blotting for the indicated proteins. Asterisk $\left({ }^{\star}\right)$ marks the IgG heavy chain. Note the lower abundance of Plk1 in asynchronous lysates. (C, top panel) Electrophoretic mobility of endogenous BubR1 from nocodazolearrested and Eg5- or Plk1-depleted HeLa cells, as determined by Western blotting. Efficient depletion of Eg5 and Plk1 and equal loading ( $\alpha$-tubulin) are shown in the panels below. (D) Pulldowns from mitotic HeLa lysate using immobilized GST-PBD ${ }^{\mathrm{WT}}$ and GST-PBD ${ }^{\mathrm{AA}}$ were probed for BubR1 (top panel) and GST (middle panel). Equal input is demonstrated by BubR1 Western blotting onto TCLs (bottom panel). (E) Kinase assay and Far Western. Substrates and kinases were incubated together in an in vitro kinase assay as described in Materials and Methods. Samples were resolved by SDS-PAGE, transferred onto nitrocellulose membrane, probed with GST-PBD ${ }^{\mathrm{WT}}$ (top panel), and then reprobed with GST-PBD ${ }^{\mathrm{AA}}$ (second panel). Notably, sequential phosphorylation by $\mathrm{Cdk} 1$ and Plk1 caused a more diffused pattern of PBD binding, suggesting a retarded mobility of BubR1, likely caused by phosphorylation. Equal levels of BubR1 (third panel) and Plk1 (bottom panel) are indicated by Western blotting. The numbers below the top panel indicate band intensities, as quantified using ImageJ software. $(F)$ Spot arrays of peptides representing the PBD-binding motif surrounding Thr 620 of BubR1 (T620), an unrelated serine/threonine-rich sequence of BubR1 (S534), and the poloboxtide, each synthesized in the nonphosphorylated (-P) and phosphorylated (+P) form. Membranes were probed with GST-PBD ${ }^{\mathrm{WT}}$ (left panel) or GST-PBD ${ }^{\mathrm{AA}}$ (right panel). Peptide sequences are described in the Supplemental Material.

examined the ability of GST-PBD ${ }^{\mathrm{WT}}$ or GST-PBD ${ }^{\mathrm{AA}}$ to bind to immobilized 12-mer peptides surrounding T620, an unrelated S/T sequence from BubR1 (S534), and the consensus Poloboxtide (Elia et al. 2003), all synthesized in their unphosphorylated and phosphorylated forms, respectively. Whereas GST-PBD ${ }^{\mathrm{WT}}$ bound strongly to the peptide harboring the phosphorylated T620 BubR1 motif, virtually no binding was seen to the same peptide in the unphosphorylated form or to the unrelated phosphopeptide centered on S534 (Fig. 1F, left panel). Similarly, the $\mathrm{PBD}^{\mathrm{WT}}$ bound to the phosphorylated, but not the unphosphorylated Poloboxtide, in agreement with previous results (Elia et al. 2003). GST-PBD ${ }^{\mathrm{AA}}$ showed virtually no binding to any of the peptides (Fig. 1F, right panel). Collectively, these results demonstrate that Cdk phosphorylation promotes PBD-mediated Plk1 docking onto BubR1, and that the evolutionarily conserved PBD consensus motif centered on T620 constitutes a likely priming site.

\section{KT localization of BubR1 and Plk1 is important for BubR1 hyperphosphorylation}

Previously, CenpE has been implicated in causing the phosphorylation-induced BubR1 upshift (Weaver et al. 2003; Tanudji et al. 2004). This prompted us to compare the effect of siRNA-mediated depletion of CenpE or Plk1 on BubR1 phosphorylation (Fig. 2A). Consistent with the results shown above, depletion of Plk1 but not Eg5 led to loss of BubR1 hyperphosphorylation. CenpE depletion also caused a decrease in BubR1 phosphorylation, in agreement with previous results (Weaver et al. 2003; Tanudji et al. 2004). This effect was not as striking as that observed for Plk1 depletion, although the depletion of CenpE was arguably not complete. Because the loss of CenpE from mouse embryo fibroblasts was reported to cause a drastic reduction in KT localization of several checkpoint components (Weaver et al. 2003), we asked whether the effect of CenpE depletion on BubR1 phos- 
Elowe et al.
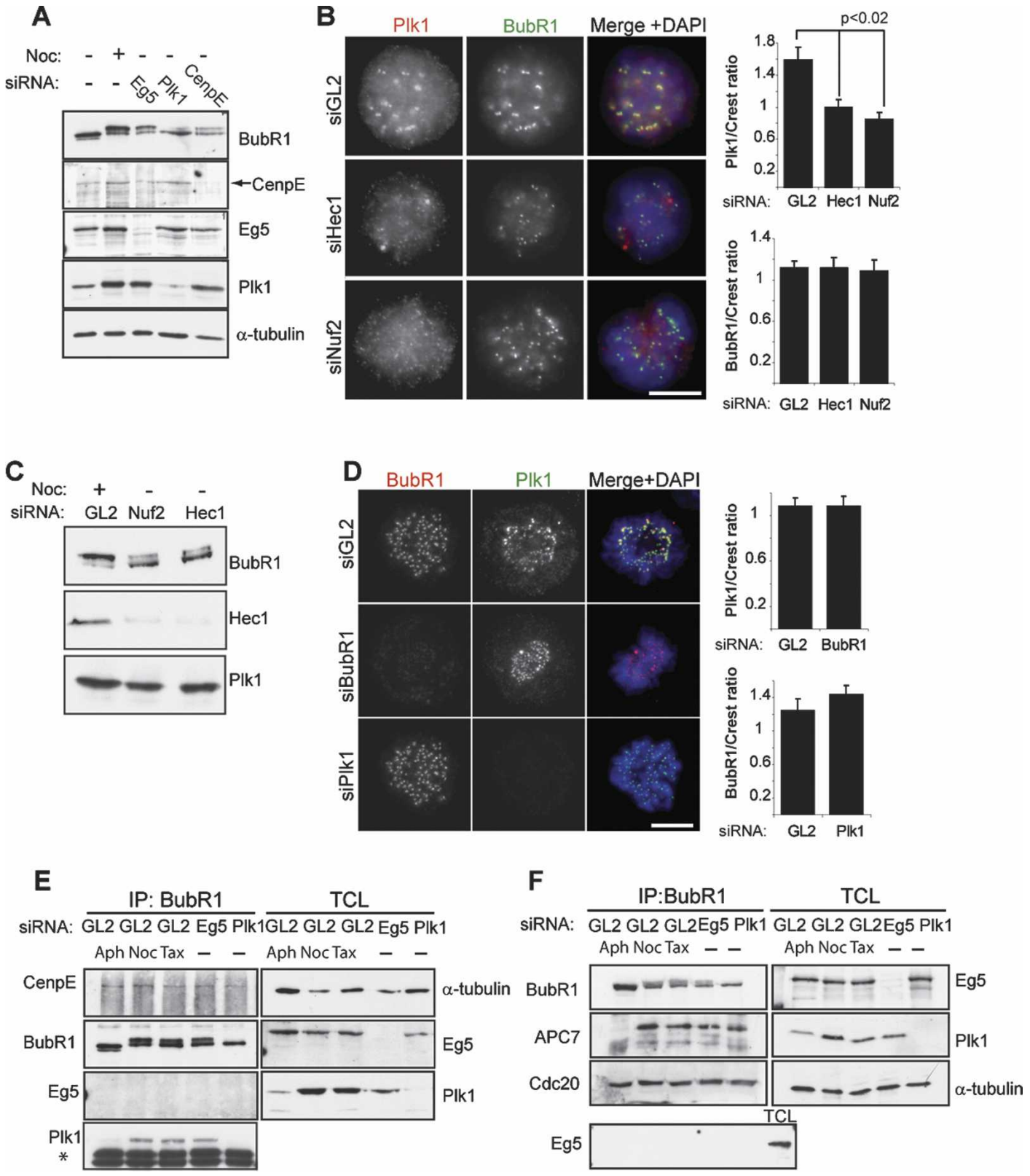

Figure 2. BubR1 hyperphosphorylation occurs primarily at the KT but does not affect BubR1 KT recruitment and SAC function. (A) Interphase lysates or mitotic lysates from nocodazole-treated and either Eg5-, Plk1-, or CenpE-depleted cells (isolated by mitotic shake-off) were resolved by gel electrophoresis. Then, the levels and migration of BubR1 (top panel), CenpE, Eg5, Plk1, and $\alpha$-tubulin were determined by Western blotting. (B) BubR1 and Plk1 localization in HeLa cells depleted of Hec1 and Nuf2. Bar, $10 \mu \mathrm{m}$. Panels on the right show quantifications of Plk1 (top) and BubR1 (bottom) staining intensities at KTs (normalized for CREST staining) in the various samples (mean \pm standard error, $\mathrm{SE} ; n \geq 100 \mathrm{KT}$ from five different cells). $(C)$ Lysates were prepared from nocodazole-arrested cells or prometaphase cells depleted of Nuf2 or Hec1. They were then probed by Western blotting with the antibodies indicated. $(D)$ Interdependence of Plk1 and BubR1 localization during mitosis was assessed by indirect immunofluorescence. Bar, $10 \mu \mathrm{m}$. Panels on the right show quantifications of Plk1 and BubR1 staining intensities at KTs (normalized for CREST staining) in reciprocal siRNA depletions (mean \pm SE; $n \geq 100 \mathrm{KT}$ s from five different cells). (E) Interphase lysates or lysates from nocodazole- or Taxol-treated as well as Eg5- and Plk1-depleted cells were prepared. (Left panels) BubR1 immunoprecipitates were then resolved by SDS-PAGE, and coimmunoprecipitation of CenpE and Plk1, as well as Eg5 (for control), was examined by Western blotting. Asterisk (*) indicates IgG heavy chain. (Right panels) In parallel, corresponding TCLs were probed by Western blotting with the antibodies indicated. $(F)$ Cell lysates were prepared as in $E$, and BubR1 immunoprecipitates were assessed for coimmunoprecipitation of APC7 and Cdc20; Eg5 was examined for control.

phorylation might be explained by mislocalization of BubR1. Indeed, whereas mock depletion had no significant effect on the KT localization of BubR1, CenpE depletion resulted in a marked reduction of BubR1 levels at KTs (Supplementary Fig. S1A), confirming previous results (Weaver et al. 2003; Tanudji et al. 2004). It is 
therefore conceivable that BubR1 phosphorylation may be attenuated in CenpE-depleted cells as a consequence of BubR1 mislocalization.

We next asked whether Plk1 mislocalization might also be sufficient to reduce BubR1 hyperphosphorylation. Depletion of either Hec1 or Nuf2, two components of the Ndc 80 complex at the outer KT, causes the mislocalization of several KT proteins (Martin-Lluesma et al. 2002; DeLuca et al. 2003; McCleland et al. 2004). Indeed, in response to Hec1 or Nuf2 depletion, Plk1 was lost from KTs, but BubR1 localization was not significantly affected (Fig. 2B). In contrast, neither Aurora B nor its kinase activity (measured by pCenpA staining) at centromeres was detectably reduced (Supplementary Fig. $\mathrm{S} 1 \mathrm{~B}, \mathrm{C})$. In line with the known interdependence of Hec1 and Nuf2 (DeLuca et al. 2003), the depletion of either protein resulted in the loss of $\mathrm{Hecl}$ (Fig. 2C, middle panel), but no significant reduction of Plk1 protein levels was observed (Fig. 2C, bottom panel). Yet, when compared with nocodazole-arrested cells, Hec1- or Nuf2-depleted cells showed a significant reduction in slowly migrating BubR1 (Fig. 2C, top panel), suggesting that robust mitotic BubR1 phosphorylation depends on Plk1 that is correctly localized to KTs. Collectively, the above results suggest that mislocalization of either BubR1 (through CenpE depletion) or Plk1 (through Hec1 or Nuf2 depletion) can lead to loss of BubR1 phosphorylation, implying that KT localization of both kinase (Plk1) and substrate (BubR1) is important for this modification to occur efficiently.

\section{BubR1 association with KT and mitotic checkpoint components are not altered upon Plk1 depletion}

To explore the functional significance of BubR1 phosphorylation by Plk1, we first asked whether BubR1 localization is influenced by Plk1 or vice versa. In cells depleted of Plk1, no significant effects on BubR1 localization were observed when compared with mock-depleted cells, indicating that neither Plk1 nor BubR1 hyperphosphorylation is required for recruitment or maintenance of BubR1 at the KT (Fig. 2D). Likewise, BubR1 depletion exerted no notable effect on Plk1 KT localization (Fig. 2D), in agreement with a recent study (Qi et al. 2006). Plk1 localization to KTs was also unaffected when BubR1-depleted cells were transfected with Flag-tagged rescuing constructs coding for either wild-type (WT) BubR1 or a BubR1 mutant (hereafter referred to as BubR1 $\left.{ }^{\mathrm{T} 620 \mathrm{~A}}\right)$, in which two residues critical for PBD binding (S619 and T620) were substituted by alanine (Supplemental Fig. S2).

As phosphorylation events often facilitate proteinprotein interactions, we next explored the possibility that BubR1 phosphorylation might be required for interactions with known binding partners. We first focused on CenpE, because a direct interaction between BubR1 and CenpE is well established (Chan et al. 1998, 1999; Abrieu et al. 2000). BubR1 was immunoprecipitated from cells depleted of either Plk1 or Eg5 (for control), as well as from aphidicolin-, nocodazole-, and Taxol-arrested cells, and the ability of BubR1 to coimmunoprecipitate CenpE was assessed. CenpE was found in BubR1 immunoprecipitates under all conditions, whereas another motor protein, Eg5, was absent (Fig. 2E). As expected, Plk1 also bound to BubR1 in mitotic samples, except when Plk1 was itself depleted. Thus, we conclude that BubR1 phosphorylation is not required for its interaction with CenpE.

BubR1 also associates with Bub3 and Cdc20 within the MCC (Sudakin et al. 2001) and with several APC/C subunits (Chan et al. 1999). Thus, we examined BubR1 immunoprecipitates for the presence of Bub3, Cdc20, and the APC/C subunit APC7. Binding of both Bub3 and Cdc20 to BubR1 was similar in all conditions (Fig. 2F; data not shown), in agreement with the stable and cell cycle-independent nature of the MCC complex (Sudakin et al. 2001). Binding of the APC/C subunit APC7 was M-phase specific, as expected, but was also not altered in Plk1-depleted cells. Together, these observations indicate that hyperphosphorylation of BubR1 is not required for its ability to bind known interaction partners, including other SAC components.

\section{Stability of KT-MT connections is compromised in cells expressing BubR1-T620A}

Loss of both BubR1 and Plk1 function has recently been proposed to perturb KT-MT connections (Sumara et al. 2004; Lampson and Kapoor 2005; Lenart et al. 2007; Matsumura et al. 2007), and several other targets of Plk1 have been implicated in maintaining KT-fiber stability (Meraldi et al. 2004; Kline-Smith et al. 2005; Kang et al. 2006; Nishino et al. 2006; Matsumura et al. 2007). To determine whether BubR1 phosphorylation by Plk1 might be required for stabilizing KT-MT interactions, BubR1-depleted cells were rescued with wild-type and various mutant BubR1 proteins, and the presence of cold-stable KT-MTs was examined using a previously described approach (Lampson and Kapoor 2005). In brief, HeLa cells were transfected simultaneously with Flagtagged BubR1 constructs and siRNA duplexes targeting the $3^{\prime}$-untranslated region (UTR) region of endogenous BubR1 transcripts (BubR1-3'), and processed as outlined schematically in Figure 3A. Rescue experiments were performed with Flag vector alone, Flag-BubR $1^{\text {WT }}$, FlagBubR1 ${ }^{\mathrm{KD}}$ (kinase dead), and the Plk1-PBD docking site mutant Flag-BubR $1^{\mathrm{T} 620 \mathrm{~A}}$. Efficiency of BubR1 depletion and the expression of each construct are demonstrated in Supplementary Figure S3A-C. In control cells, cold treatment produced the expected progressive destabilization of MTs, but did not cause a generalized loss of Plk1 from KTs (Supplementary Fig. S3D). BubR1-depleted cells cotransfected with the empty Flag vector showed only very few cold-stable MTs, and very few CREST-stained KTs had any MTs attached, in agreement with previous observations (Lampson and Kapoor 2005). In contrast, cells cotransfected with BubR1 siRNA and either FlagBubR $1^{\text {WT }}$ or Flag-BubR1 ${ }^{\mathrm{KD}}$ showed relatively intact 
A

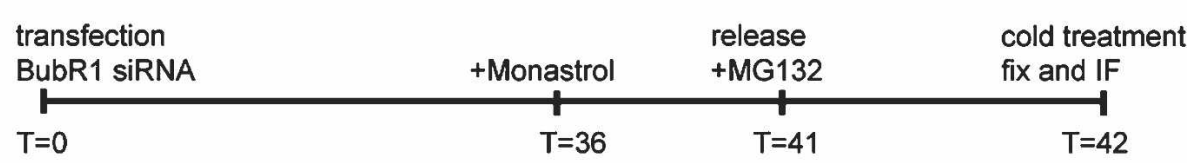

B

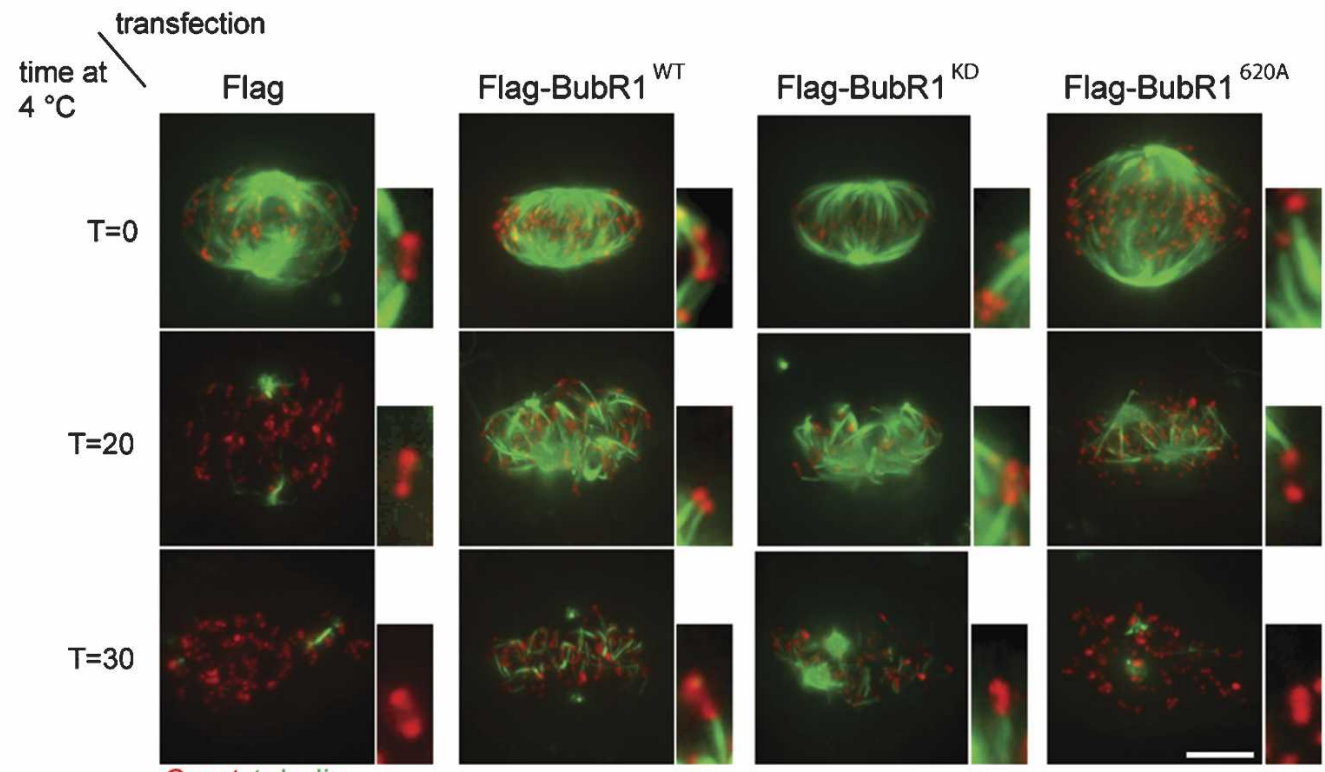

Crest tubulin

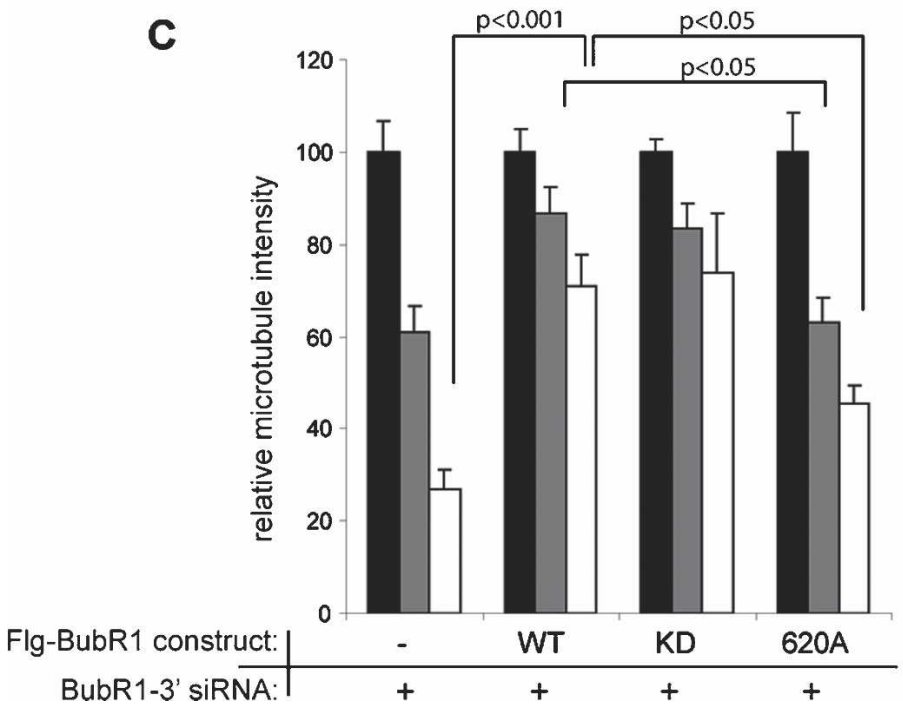

Figure 3. The cold stability of KT-MT connections is reduced in cells expressing BubR1 ${ }^{\mathrm{T} 620 \mathrm{~A}}$. (A) Schematic representation of BubR1 siRNA, rescue transfection, and cold treatment protocol. HeLa S3 cells were simultaneously transfected with the indicated Flag-BubR1 constructs and BubR1-3' duplexes. After 36 h, cells were treated with Monastrol for $5 \mathrm{~h}$, followed by a 1-h release in the presence of the proteosome inhibitor MG132, resulting in a metaphase-arrested population. Cells were then incubated for the indicated times at $4^{\circ} \mathrm{C}$ before they were fixed and processed for immunofluorescence microscopy. (B) Cells treated according to the protocol outlined in $A$ were costained for $\alpha$-tubulin (green), KTs (CREST) (red), and Flag (not shown). Bar, $10 \mu \mathrm{m}$. Representative KT-MT connections were enlarged and are shown on the right. (C) Quantification of MT density in B. Average MT intensity (mean $\pm \mathrm{SE}_{;} n \geq 10$ transfected cells) was measured in each condition. Intensities are expressed relative to total cellular areas and normalized against $\mathrm{T} 0$ for each condition (MT density at T0 $=100 \%$ ).

spindles, with most CREST-stained KTs clearly attached to $\mathrm{MT}$ fibers, even after $30 \mathrm{~min}$ at $4^{\circ} \mathrm{C}$ (Fig. 3B). Most importantly, BubR1-depleted cells expressing Flag-
BubR1 $1^{\mathrm{T} 620 \mathrm{~A}}$ exhibited markedly fewer cold-stable MTs; most KTs were unattached already after 20 min of cold treatment and completely lost after $30 \mathrm{~min}$ (Fig. 3B). 
Measurements of relative MT intensities shown in Figure 3C confirm these observations. Collectively, these data show that the impaired BubR1-Plk1 association, followed by the consequent loss of BubR1 phosphorylation by Plk1, results in reduced stability of KT-MTs.

BubR $1^{\text {T620A }}$-expressing cells show chromosome congression defects and mitotic delay

If, as our above data indicate, BubR1 phosphorylation is required for the stabilization of KT-MT attachments, then loss of the BubR1-Plk1 interaction should result in defects in chromosome congression. Thus the ability of WT, KD, and T620A BubR1 to compensate for BubR1 depletion during chromosome congression was examined by time-lapse videomicroscopy. To visualize transfected cells, all BubR1 constructs were generated with an $\mathrm{N}$-terminal fluorescent mCherry tag (Shaner et al. 2004). They were then transfected together with BubR1-3' siRNA duplexes into HeLa cells stably expressing GFPtagged histone $\mathrm{H} 2 \mathrm{~B}$, and imaged as indicated in Figure 4A (see Supplementary Movies S1-S4). Representative stills from each movie, taken at the indicated time points after the onset of chromosome condensation, are shown in Figure 4B. BubR1-depleted cells expressing only the mCherry tag rapidly exited mitosis without forming a metaphase plate, as expected (Chan et al. 1999; Shannon et al. 2002). Rescue of BubR1-depleted cells with either mCherry-tagged BubR $1^{\mathrm{WT}}$ or BubR $1^{\mathrm{KD}}$ re-

A

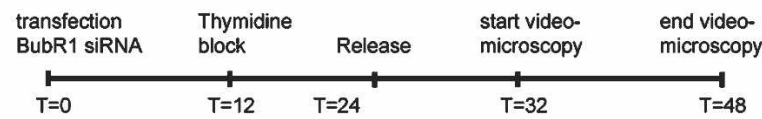

B Cherry Chromosome

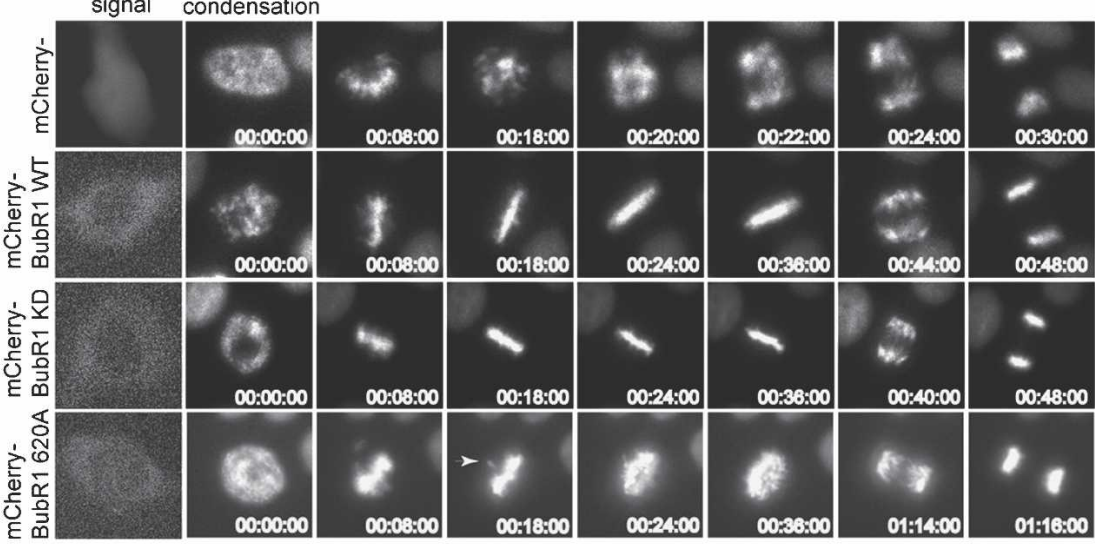

C

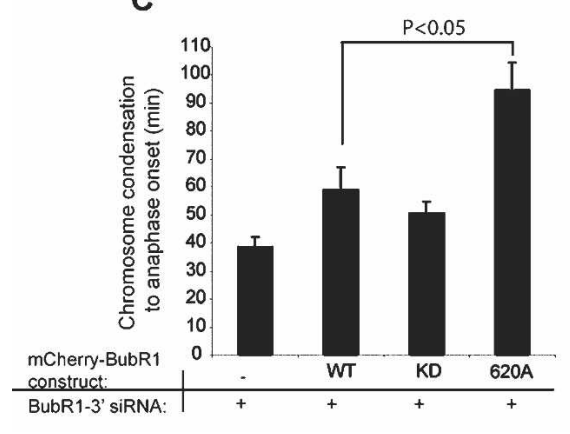

D

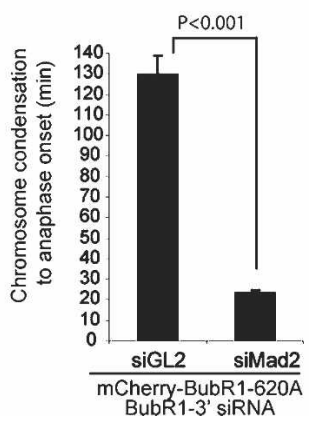

Figure 4. Time-lapse videomicroscopy of cells expressing BubR1 WT, KD, or T620A. (A) Schematic representation of BubR1 siRNA, rescue transfection, and videomicroscopy protocol. Cells were simultaneously transfected with the indicated mCherry-BubR 1 constructs and BubR1-3' duplexes, and arrested by addition of thymidine $12 \mathrm{~h}$ later. After a further $12 \mathrm{~h}$, they were released from the block, and imaging was started $8 \mathrm{~h}$ later for a total duration of $16 \mathrm{~h} .(B)$ Representative stills illustrating mitotic progression in cells depleted of BubR1 and rescued with mCherry-BubR1 constructs. Images were acquired at the indicated time points after the start of chromosome condensation. $(C)$ Histogram indicating the time elapsed between the beginning of chromosome condensation and anaphase onset in cells treated as in $A$. Results present the average of five independent experiments $( \pm \mathrm{SE} ; n \geq 8$ cells per experiment). $(D)$ As in $C$, except that cells expressing mCherry-BubR $1^{\mathrm{T} 620 \mathrm{~A}}$ were transfected with $3^{\prime}$-UTR siRNA duplexes against BubR1 and either GL2 or Mad2. Results present the average of four independent experiments $( \pm \mathrm{SE} ; n \geq 34$ cells each condition). 
sulted in cells showing proper chromosome congression to a metaphase plate, followed by timely onset of anaphase (Fig. 4B). In stark contrast, cells depleted of endogenous BubR1 but expressing mCherry-BubR $1^{\mathrm{T} 620 \mathrm{~A}}$ showed aberrant congression, characterized by slow chromosome capture (Fig. 4B, see arrowhead in 18:00 panel) and the formation of broad metaphase plates. BubR1 $1^{\text {T620A }}$-expressing cells also exhibited prolonged mitoses, requiring on average almost twice as long as BubR1 ${ }^{\mathrm{WT}}$ - or BubR1 ${ }^{\mathrm{KD}}$-expressing cells to reach anaphase (Fig. 4C). Importantly, there were no precocious anaphases observed in BubR1 $1^{\mathrm{T} 620 \mathrm{~A}}$-expressing cells, suggesting that these cells were able to sustain an active spindle checkpoint. This was confirmed by transfecting BubR1-depleted cells expressing BubR1 ${ }^{\mathrm{T} 620 \mathrm{~A}}$ with an siRNA duplex depleting Mad2, which was expected to abrogate the SAC. Under these conditions, cells exited mitosis rapidly, without achieving proper metaphase (Fig. 4D).

\section{Identification of S676 as an in vivo Plk1} phosphorylation site in BubR1

We next sought to identify mitosis-specific in vivo phosphorylation sites on BubR1. Mass spectrometric analysis of mitotic spindles identified S676 as one prominent phosphorylation site on BubR1 (Fig. 5A). Mitosis-specific phosphorylation was confirmed using a SILAC approach (Mann 2006), which demonstrated a threefold up-regulation of phosphorylation at this residue in nocodazole versus thymidine-arrested extracts (Fig. 5B). Closer examination of S676 and the surrounding sequence in BubR1 from different organisms revealed that this residue lies within an evolutionarily conserved Plk1 consensus phosphorylation motif (Fig. 5C), suggesting that it represents an important in vivo Plk1 site on BubR1. Therefore, a polyclonal antibody directed against a peptide encompassing pS676 was generated. The specificity of immune versus preimmune sera for phospho-BubR1 was verified by costaining with BubR1 (Supplementary Fig. S4A) and Western blotting on phosphatase-treated mitotic extracts (Supplementary Fig. S4B). By Western blotting on whole-cell lysates, the anti-pS676 antibody recognized a single band with an apparent molecular weight of $130 \mathrm{kDa}$ in mitotic but not interphase samples (Fig. 6A, left panel). Reprobing the membrane for BubR1 demonstrated that this band corresponds exactly to the slowly migrating, hyperphosphorylated form of BubR1 (Fig. 6A, top right panel). Anti-pS676 reactivity to this band was lost upon phosphatase treatment, confirming its specificity for phosphorylated BubR1 (Supplementary Fig. S4B). Most importantly, anti-pS676 identified the hyperphosphorylated BubR1 band in lysates from nocodazole-arrested and Eg5-depleted cells, but not from interphase or Plk1-depleted cells (Fig. 6B). Entirely consistent results were also obtained by immunofluorescence microscopy. Whereas control and Eg5-depleted cells exhibited strong KT staining with the anti-pS676 antibody, cells depleted of either Plk1 or BubR1 (using either one of
A

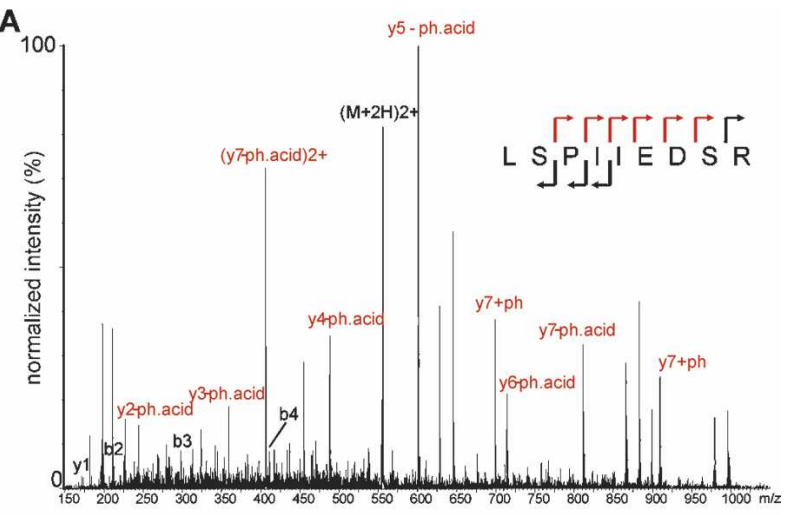

B

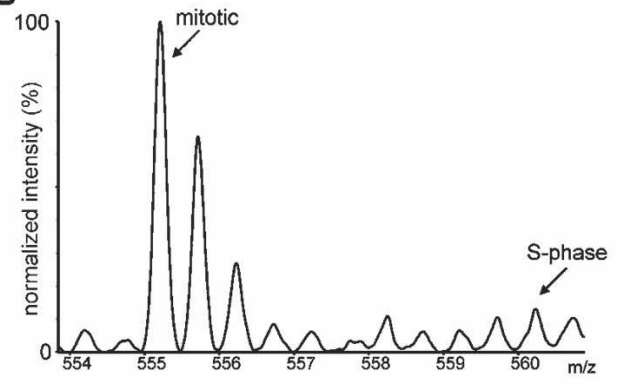

phosphopeptide ratio (M/S) uncorrected: $>5.3$

protein level ratio (M/S):1.82

phosphopeptide ratio (M/S) (corrected): $>2.9$

C
Homo sapiens
Mus musculus
Gallus gallus
Xenopus laevis
Danio rerio*

Figure 5. Identification of Ser 676 as an in vivo phosphorylation site on BubR1. (A) Collision-induced dissociation (CID) mass spectrum of the BubR1-derived phosphopeptide LSPIIED(pS)R. C-terminal and N-terminal fragments of the peptide are marked as y-ions and b-ions, respectively. Fragments containing the phosphate are marked in red, and fragments showing a characteristic loss of phosphoric acid are labeled "-ph.acid." The observed peptide fragments are also shown within the peptide sequence above the spectrum, demonstrating that the indicated serine residue within this peptide is indeed phosphorylated. This spectrum was acquired on a quadrupole time-of-flight mass spectrometer (Q-TOF Ultima). (B) The relative intensity of the phosphopeptide shown in $A$ was measured in a mitotic versus an S-phase-arrested extract, using stable isotope labeling in cell culture (see Materials and Methods). The observed ratio was corrected to compensate for the higher expression level of BubR1 in mitosis compared with $\mathrm{S}$ phase, as determined using unphosphorylated BubR1-derived peptides from the same sample. (C) Sequence alignment of S676 (red box) and surrounding residues indicating conservation of this site in several BubR1-expressing species. Asterisk $\left({ }^{\star}\right)$ indicates sequence predicted by bioinformatics data mining.

two independent siRNA duplexes) showed no staining (Fig. 6C and quantification in E). Specificity of this antibody was further confirmed in rescue experiments with 

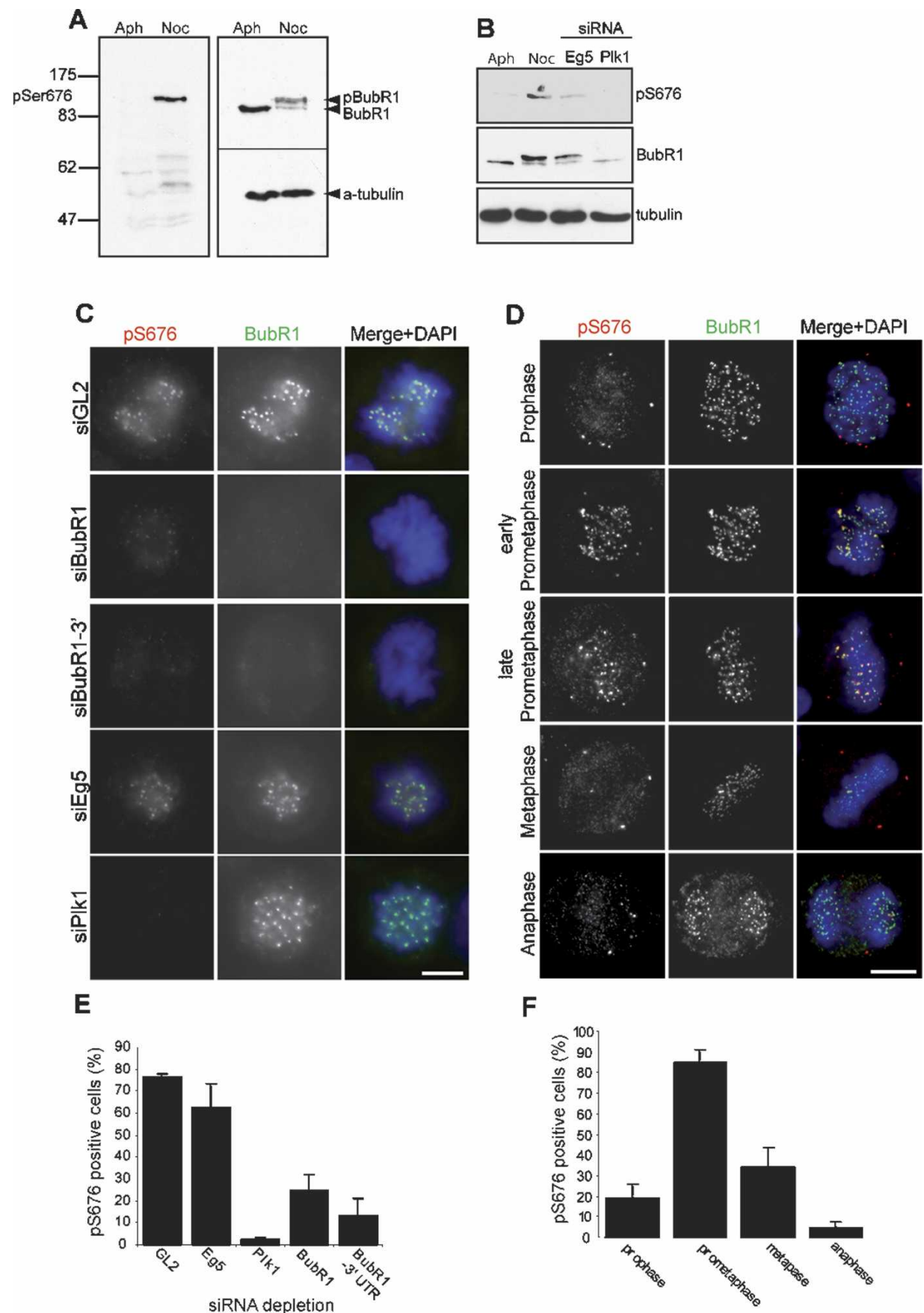

F

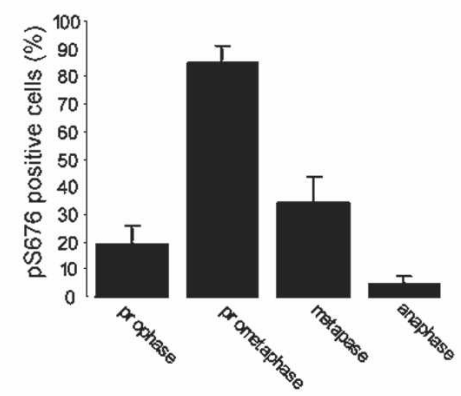

Figure 6. BubR1 Ser 676 is a Plk1-dependent phosphorylation site. (A) Anti-pS676 was used in Western blotting on lysates from aphidicolin-arrested (Aph) and nocodazole-arrested (Noc) cells. The same blot was stripped and reprobed with anti-BubR1 antibodies (top right panel) and tubulin (bottom right panel). (B, top panel) Western blotting with anti-pS676 onto aphidicolin-arrested (Aph) and nocodazole-arrested (Noc) cells, and onto cells depleted of Eg5 and Plk1 by siRNA. The membrane was reprobed with anti-BubR1 antibodies (middle panel), and equal loading is demonstrated by Western blotting for $\alpha$-tubulin (bottom panel). (C) Immunofluorescent staining of cells treated with the indicated siRNA duplexes. Cells were costained with anti-pS676 (red), anti-BubR1 (green), and DAPI (blue). Bar, $10 \mu \mathrm{m}$. (D) Mitotic cells within an asynchronously growing HeLa cell population were costained for pS676 (red) and BubR1 (green). DNA was stained with DAPI (blue). Bar, $10 \mu \mathrm{m} .(E, F)$ Quantification of the results in $C$ and $D$, respectively. Data represent the mean percentage of cells staining positive for pS676 immunofluorescence on most if not all KTs (mean \pm SE; $n \geq 100$ cells for each of three independent experiments).

Flag-BubR1 WT, KD, or T620A. Whereas BubR1-depleted cells rescued by BubR $1^{\mathrm{WT}}$ and BubR $1^{\mathrm{KD}}$ stained strongly with the anti-pS676 antibody, cells expressing
BubR $1^{\mathrm{T} 620 \mathrm{~A}}$ showed little or no staining (Supplementary Fig. S4C and quantification in D). These results demonstrate that T620 is, indeed, the in vivo binding site for 
Plk1 and that abrogating the BubR1-Plk1 association abolishes phosphorylation of BubR1 at S676.

Having established the specificity of the anti-pS676 antibody, this reagent was used to trace the timing of BubR1 phosphorylation by Plk1. Analysis of asynchronously growing HeLa cells by immunofluorescence microscopy revealed that S676 phosphorylation was observed mainly in prometaphase, when the majority of cells showed strong KT staining. Phosphorylation was gradually lost as chromosomes congressed so that fully aligned metaphase plates showed little or no phosphorylation, and almost no anaphase cells were found to stain positive with the anti-pS676 antibody (Fig. 6D and quantification in F). Taken together, these results demonstrate that S676 on BubR1 represents a bona fide prometaphase-specific in vivo target of Plk1 and that phosphorylation of this residue occurs predominantly during prometaphase, when MT attachments are established.

\section{BubR1 phosphorylation at S676 occurs upon lack of tension}

The observation that pS676 staining was lost concomitant with the alignment of chromosomes at the metaphase plate suggested that loss of S676 phosphorylation correlates with the establishment of tension between sister KTs. In support of this view, the anti-pS676 antibody strongly stained the KTs of misaligned chromosomes in CenpE-depleted cells, but not those of chromosomes already aligned at a metaphase plate (Fig. 7A). To further test the dependency of S676 phosphorylation on the lack of tension, HeLa cells were arrested at metaphase by MG132 treatment and then exposed to Taxol, which abolished the already established tension (Nishino et al. 2006). DMSO-treated control cells exhibited metaphase plates with clear BubR1 staining, but no notable pS676 signal, indicating that KTs were under tension (Fig. 7B). However, even short treatment with Taxol led to the rapid reappearance of a strong pS676 signal at metaphase plates (Fig. 7B, and quantification in C). To exclude the possibility that Taxol caused a loss of the pS676 signal due to epitope masking, we also assayed S676 phosphorylation by Western blotting. As expected, anti-pS676 identified the upshifted BubR1 band in lysates from nocodazole-treated but not asynchronously growing HeLa cells (Fig. 7D). Similarly, and in full agreement with our immunofluorescence results, pS676 blotting also identified hyperphosphorylated BubR1 in extracts from Taxol-treated cells, whereas no pS676 signal was detected in MG132-arrested cells, concomitant with reduced BubR1 hyperphosphorylation (Fig. 7D). High cyclin B1 levels confirm the mitotic state of the lysates, and Western blotting for tubulin demonstrates equal loading. Taken together, these data illustrate that BubR1 phosphorylation at S676 occurs in response to lack of tension. This may reflect regulation of Plk1, a counteracting phosphatase (or both), or a change in the substrate.

Having shown that Plk1 phosphorylation of BubR1 is necessary for the stability of KT-MT attachments (Figs.
$3,4)$ and considering that lack of tension destabilizes KT-MT interactions, we next asked whether $\mathrm{S} 676$ phosphorylation is also indicative of reduced KT-MT stability. As a marker for KT-MT stability, we monitored KT localization of RanGAP1, the activating protein for the small GTPase Ran, whose KT localization is known to require stable KT-MT interactions (Joseph et al. 2004). DMSO-treated cells with metaphase plates showed the expected spindle and KT staining for RanGAP1, with little or no pS676 staining (Fig. 7E). In contrast, in Taxoltreated cells, RanGAP1 staining became diffuse, indicating that the protein was largely lost from the KTs, whereas pS676 staining at KTs was drastically increased. Examination of the RanGAP1 versus pS676 signal from individual KTs in a number of cells reveals an inverse relationship between RanGAP1 KT recruitment and BubR1 phosphorylation at S676 (Fig. 7F). These results confirm that BubR1 phosphorylation correlates with the loss of KT-MT stability that accompanies the lack of tension.

\section{Discussion}

In this study, we have addressed the cause and function of the characteristic mitotic hyperphosphorylation of BubR1. We have identified Plk1 as the kinase responsible for this hyperphosphorylation and the highly conserved Ser 676 as the first in vivo-verified M-phase-specific phosphorylation site on BubR1. Using a phospho-specific antibody raised against pS676, we show that this reagent can be used to monitor tension between sister KTs. Loss of the BubR1-Plk1 interaction and the ensuing loss of BubR1 hyperphosphorylation did not detectably interfere with KT localization of either Plk1 or BubR1, nor with the ability of BubR1 to associate with CenpE, MCC components, or the APC/C. Rather than being directly required for SAC signaling, Plk1-induced BubR1 hyperphosphorylation was found to be important for the establishment of stable KT-MT interactions during chromosome congression. Remarkably, phosphorylation of S676 was prominent on all KTs during prometaphase and on unaligned chromosomes in CenpE-depleted cells. Moreover, it could be restored to metaphase chromosomes by Taxol treatment, demonstrating that this modification on BubR1 correlates with the extent of tension between sister KTs.

\section{Plk1 phosphorylation causes the BubR1 mitotic upshift}

Two recent studies independently confirm that Plk1 is responsible for the mitotic hyperphosphorylation that causes the characteristic upshift in BubR1 migration (Lenart et al. 2007; Matsumura et al. 2007). However, previously two other proteins had been implicated in causing this BubR1 upshift, namely, CenpE and Aurora B (Ditchfield et al. 2003; Weaver et al. 2003; Tanudji et al. 2004). Interestingly, depletion of CenpE results in decreased BubR1 levels at KTs (Supplementary Fig. S1; 
A
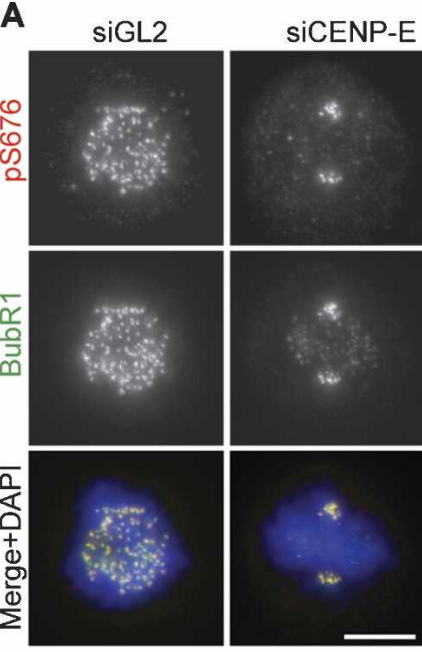

B
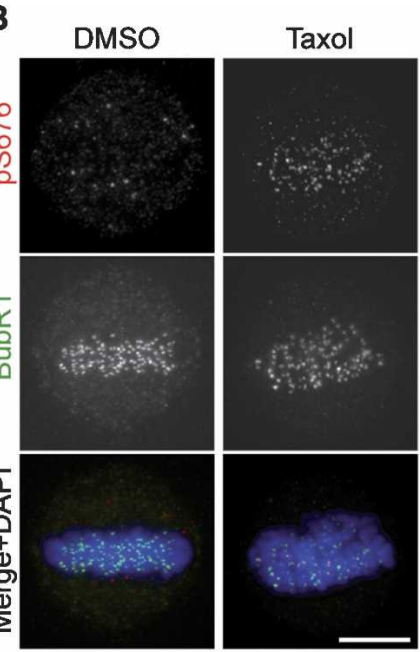

C
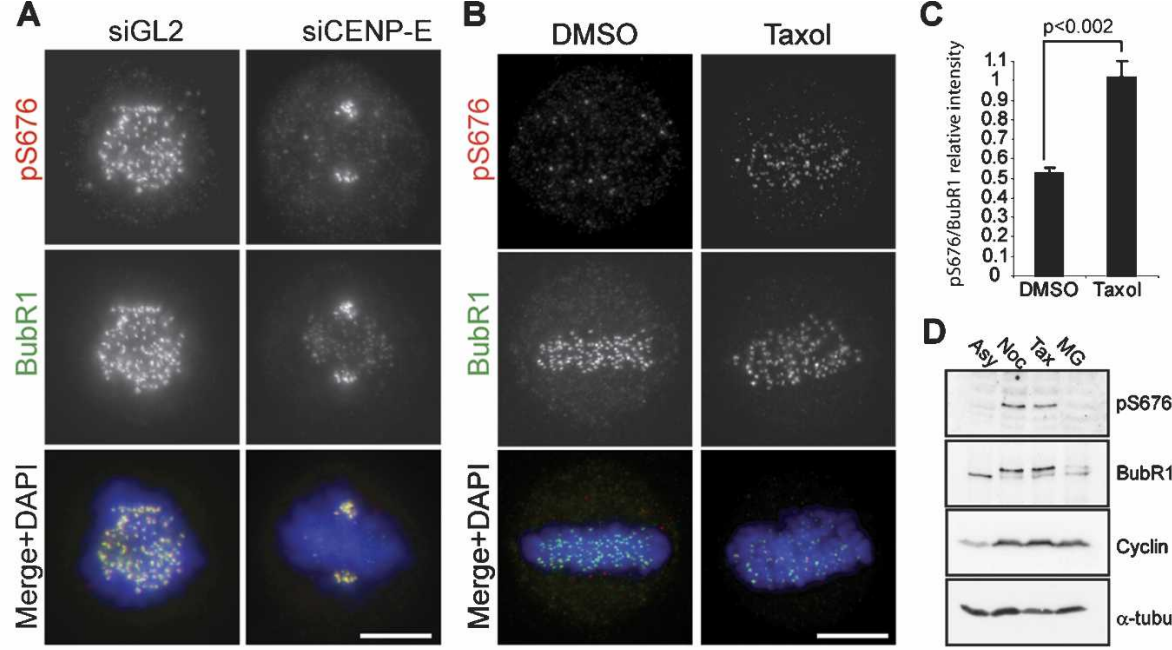

D
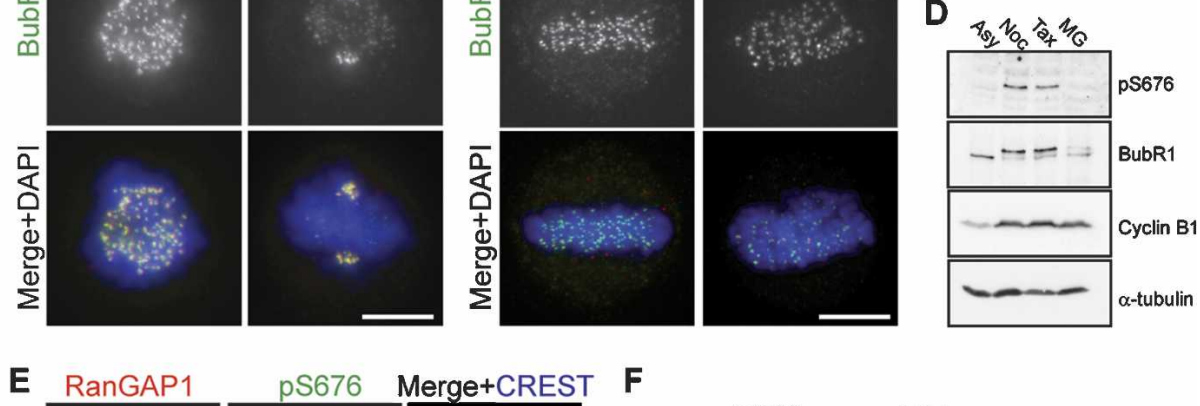

pS676 Merge+CREST

\section{$F$}
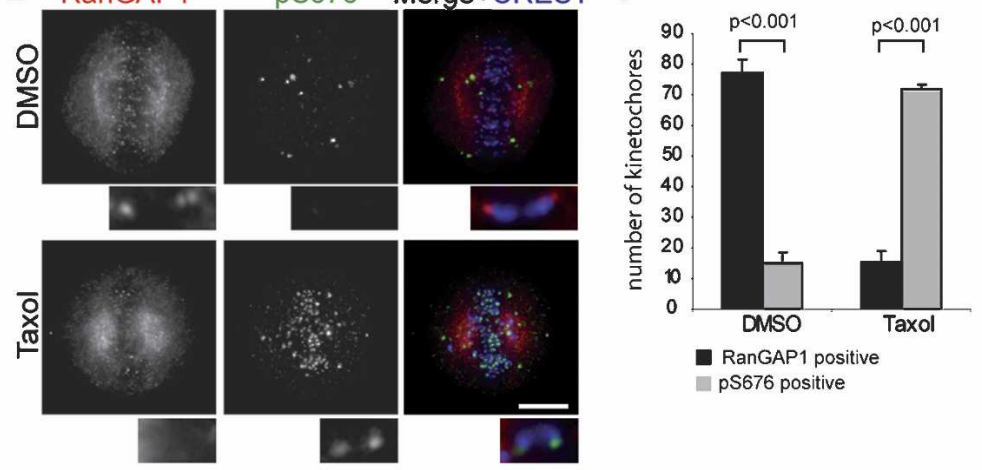

G

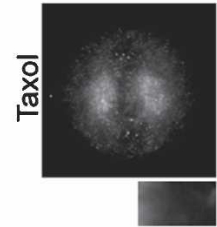

PROPHASE
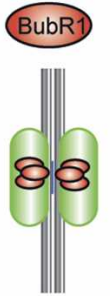

BubR1 S676 unphosphorylated No KT-MT interactions

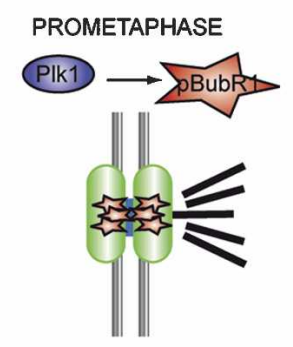

BubR1 S676 phosphorylated Establishment of KT-MT interactions

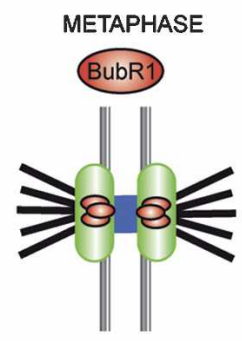

BubR1 S676 no longer phosphorylated

Stable KT-MT interactions and tension established

Figure 7. Ser 676 is phosphorylated on misaligned KTs and in response to lack of tension. (A) Control-depleted (siGL2) or CenpEdepleted (siCenpE) cells were stained with anti-pS676 (red) and anti-BubR1 (green) antibodies. DNA is visualized with DAPI (blue). (B) Cells were blocked in prometaphase using Monastrol $(16 \mathrm{~h})$ and subsequently released for $1 \mathrm{~h}$ into MG132 to allow for metaphase plate formation. Parallel samples were then treated for $1 \mathrm{~h}$ with $10 \mu \mathrm{M}$ Taxol to induce loss of tension or DMSO for solvent control. Cells were fixed and costained for pS676 (red) and BubR1 (green). DNA was visualized using DAPI (blue). Bar, $10 \mu \mathrm{m}$. (C) Quantification of $B$. Intensity ratios of pS676 normalized for total BubR1 (mean \pm SE; $n \geq 100 \mathrm{KT}$ srom five individual cells). (D) Lysates were prepared from asynchronously growing cells (Asy), nocodazole-arrested cells (Noc), or Taxol-arrested cells (Tax), and cells arrested at metaphase by MG132 treatment after release from prometaphase block (MG). (Top panel) They were resolved by SDS-PAGE and probed by Western blotting for pS676. The same membrane was subsequently reprobed for total BubR1, cyclin B1, and $\alpha$-tubulin. (E) Cells were treated as in B and then costained for RanGAP1 (red), pS676 (green), and CREST (blue). Selected KT-MT attachments were enlarged and are shown below each image. Bar, $10 \mu \mathrm{m} .(F)$ KTs from $E$ were examined for the presence of RanGAP1 and pS676 signals after DMSO or Taxol treatment. The histogram shows mean KT numbers $\left( \pm \mathrm{SE}_{;} n>500 \mathrm{KT}\right.$ s from eight individual cells). (G) Working model describing the proposed role of BubR1 phosphorylation (see Discussion). 
Weaver et al. 2003; Tanudji et al. 2004), and similar effects were seen after depletion or inhibition of Aurora B (Ditchfield et al. 2003). Considering that maximal BubR1 hyperphosphorylation depends on KT localization of both BubR1 and Plk1 (Fig. 2A-C), impaired BubR1 localization might thus contribute to explain the reduced hyperphosphorylation seen in response to depletion of either CenpE or Aurora B. This by no means excludes the possibility that Aurora B phosphorylates mammalian BubR1. Studies in Saccharomyces cerevisiae clearly indicate that Mad3p, the putative yeast ortholog of BubR1, is phosphorylated by both Ipl1 (Aurora B) and Cdc5 (Plk1) (Rancati et al. 2005), and phosphorylation of Mad3p by Ipl1 has been implicated in the cellular response to tension (King et al. 2007). However, the Ipll sites identified in yeast Mad3p are not obviously conserved in human BubR1 and, at least under the in vitro assay conditions used here, Plk1 was much more potent in phosphorylating BubR1 than Aurora B. This notwithstanding, it will be interesting to explore a direct action of Aurora B on BubR1, particularly since vertebrate Aurora B is thought to promote turnover of KT-MTs to reduce attachment errors (Hauf et al. 2003; Cimini et al. 2006).

\section{BubR1 phosphorylation is not required for SAC signaling}

BubR1 from Plk1-depleted cells could bind to all interaction partners examined (Fig. 2E,F), indicating that BubR1 phosphorylation by Plk1 is not required for its association with MCC components or the APC/C. Moreover, BubR $1^{\mathrm{T} 620 \mathrm{~A}}$-expressing cells did not commence anaphase before all chromosomes were fully aligned (Fig. $4 \mathrm{~B}, \mathrm{C})$, indicating that the SAC was still functional. Indeed, the SAC was previously shown to be active in Plk1-depleted cells, with checkpoint proteins still associated with KTs (Sumara et al. 2004; van Vugt et al. 2004; Ahonen et al. 2005). It follows that BubR1 phosphorylation by Plk1 is not required for SAC activity per se, in line with a recent report by Nishida and coworkers (Matsumura et al. 2007).

In contrast to the striking phenotypes observed in BubR1-depleted cells expressing BubR1 ${ }^{\mathrm{T} 620 \mathrm{~A}}$, we were unable to detect significant differences in the abilities of BubR $1^{\mathrm{WT}}$ and BubR1 ${ }^{\mathrm{KD}}$ to restore BubR1 functionality in two independent assays. However, an independent study recently reported that BubR1 kinase activity is required for stabilizing KT-MT interactions (Matsumura et al. 2007). Although we cannot presently explain the discrepancy between our results and those reported by Nishida and coworkers, we propose that BubR1 activity is either not required for the functions that were assayed in the present study or, alternatively, that restoration of a catalytically inactive BubR1 to BubR1-depleted cells somehow enhanced the functionality of residual wildtype BubR1. In any event, there is evidence that BubR1 kinase activity may not be critical for all BubR1 functions. First, BubR $1^{\mathrm{KD}}$ or BubR1 lacking the kinase domain was reported to be functional in Xenopus egg extracts (Chen 2002). Second, the putative budding yeast ortholog of BubR1, Mad3, lacks a kinase domain altogether. The fact that Mad3 is also a physiological Plk1 substrate (Rancati et al. 2005) supports the view that Plk1 regulates functions of BubR1/Mad3 that do not involve a catalytic kinase domain.

\section{Plk1 phosphorylates BubR1 in response to lack of tension}

Increasing evidence suggests that Plk 1 is required for stabilization of KT-MT attachments (Sumara et al. 2004; Hanisch et al. 2006; Lenart et al. 2007; Matsumura et al. 2007). Our present data indicate that Plk1 contributes to regulate attachment stability at least in part through its association with and phosphorylation of BubR1 at the $\mathrm{KT}$, in agreement with Nishida and colleagues (Matsumura et al. 2007). Expression of BubR $1^{620 A}$ (which cannot bind to Plk1 and thus cannot become phosphorylated by Plk1) resulted in reduced cold stability of KT-MT attachments, chromosome congression defects, and prolonged mitoses (Figs. 3, 4). In addition to BubR1, Plk1 phosphorylates other KT components implicated in the establishment of KT-MT attachments (Meraldi et al. 2004; Kline-Smith et al. 2005; Kang et al. 2006; Nishino et al. 2006). Furthermore, KT binding of Plk1 and Hec1/ Ndc80, a key regulator of KT-MT attachments (Cheeseman et al. 2006; DeLuca et al. 2006), is seemingly interdependent, suggesting that these proteins may act synergistically to regulate KT-MT attachments (Ahonen et al. 2005; Wong and Fang 2005). Therefore, it is likely that Plk1 functions through cooperative phosphorylation of several targets at the KT.

Tension-sensitive phosphorylation at the KT was discovered more than a decade ago, thanks largely to the use of the 3F3/2 antibody (Gorbsky and Ricketts 1993). Although Plk1 has been implicated in generating the tension-related 3F3/2 phospho-epitope (Ahonen et al. 2005; Wong and Fang 2005) and several antigens have been tentatively linked to this epitope (Daum and Gorbsky 1998; Daum et al. 2000; Wong and Fang 2006), the significance of various phosphorylation events for KT function and tension sensing remains to be understood. Here we describe the identification of S676 as a highly conserved Plk1-specific target site on BubR1. Several lines of evidence indicate that phosphorylation of this site correlates with lack of tension. First, KTs stained with antipS676 antibody preferentially during prometaphase. Staining was gradually lost as sister chromatids reached metaphase, concomitant with the generation of tension in response to bipolar attachment (Pinsky and Biggins 2005). Second, prometaphase cells were equally phosphorylated on S676 in the presence of Taxol or nocodazole, indicating that loss of tension rather than lack of attachment efficiently induced phosphorylation (Fig. 7D; Supplementary Fig. S4E). Third, BubR1 phosphorylation was enhanced at KTs of misaligned chromosomes in CenpE-depleted cells (Fig. 7A), presumed to be attached but not under tension (Yao et al. 2000; McEwen et al. 2001). Fourth, Taxol treatment of metaphase cells led to rapid reacquisition of the anti-pS676 signal and concomi- 
tant loss of RanGAP1 binding to KTs (Fig. 7B,C), indicative of destabilized KT-MT interactions due to loss of tension (Joseph et al. 2004). These results raise the question of how pS676 relates to the 3F3/2 phospho-epitope. A recent study in Xenopus suggested that loading of the $3 \mathrm{~F} 3 / 2$ epitope onto KTs is dependent on prior assembly of checkpoint proteins and that simultaneous recruitment of BubR1 and Plk1 represents the final step in KT loading of the $3 \mathrm{~F} 3 / 2$ antigen. Although these observations made it tempting to speculate that pS676 could make a major contribution to the $3 \mathrm{~F} 3 / 2$ epitope, preliminary experiments have failed to substantiate this possibility (J. Daum, K. Byrd, and G. Gorbsky, pers. comm.).

At present, we do not know the exact biochemical role of phosphorylation of BubR1 on S676 (and presumably other Plk1 sites), but we emphasize that this phosphorylation is transient during M-phase progression, as summarized in Figure 7G. In prophase, before MTs approach KTs, there is virtually no phosphorylation at S676, which is in striking contrast to $3 \mathrm{~F} 3 / 2$ staining and indicates that $\mathrm{S} 676$ phosphorylation requires KT-MT interactions. As cells progress through prometaphase, KTs begin to associate with spindle MTs, but as long as sister chromosomes have not undergone bipolar attachment, tension is absent and KT-MT interactions are unstable. At this stage, S676 on KT-associated BubR1 is maximally phosphorylated by Plk1, and we postulate that the highly phosphorylated BubR1 contributes to the establishment of stable KT-MT interactions during chromosome congression. However, as soon as KT-MT interactions come under tension (due to bipolar attachment), phosphate is lost from S676. We thus propose that transient phosphorylation of BubR1 on S676 (and perhaps other Plk1 sites) is required for stabilizing KT-MT interactions. Whether this involves a conformational change in BubR1 or the recruitment of additional proteins remains to be determined in the future. Finally, it will be interesting to explore whether this tension-sensitive phosphorylation is regulated primarily at the level of the substrate, the upstream kinase (Plk1), or an as-yet-unidentified antagonistic phosphatase.

\section{Materials and methods}

Cell culture, synchronizations, transfections, and siRNA depletion

HeLa S3 cells or HeLa S3 cells stably expressing Histone H2B-GFP were routinely maintained in DMEM (Invitrogen) supplemented with $10 \%$ FBS and penicillin-streptomycin (100 $\mathrm{IU} / \mathrm{mL}$ and $100 \mathrm{mg} / \mathrm{mL}$, respectively). Methods for plasmid transfections and siRNA depletions as well as siRNA duplexes were previously described (Baumann et al. 2007). BubR1-specific siRNA duplexes (BubR1 [5'-GGAGATCCTCTA CAAAGGG-3'] and BubR1-3' directed against the $3^{\prime}$-UTR region [5'-GTCTCACAGATTGCTGCCT-3']) were purchased from Qiagen. Drug treatments of cells were performed for $16 \mathrm{~h}$, using aphidicolin at $1.6 \mu \mathrm{g} / \mathrm{mL}$, nocodazole at $0.5 \mu \mathrm{g} / \mathrm{mL}$ and Taxol at $10 \mu \mathrm{M}$, and MG132 at $10 \mu \mathrm{M}$ for $3 \mathrm{~h}$, or as otherwise stated.

\section{Biochemical assays}

In vitro phosphorylation of BubR 1 was carried out at $30^{\circ} \mathrm{C}$ using $300 \mathrm{ng}$ of recombinant BubR1 and $100 \mathrm{ng}$ of each kinase in a total volume of $30 \mu \mathrm{L}$ of kinase reaction buffer $(10 \mathrm{mM}$ Tris at $\mathrm{pH} 7.5,10 \mu \mathrm{M}$ sodium vanadate, $10 \mathrm{mM} \mathrm{MgCl} 2,10 \mu \mathrm{M}$ ATP, 1 mM DTT, $5 \mu \mathrm{Ci}\left[\gamma_{-}{ }^{32} \mathrm{P}\right]$ ATP $\left.[3000 \mathrm{Ci} / \mathrm{mmol}, 10 \mathrm{mCi} / \mathrm{mL}]\right)$. Reactions were stopped after $30 \mathrm{~min}$ by addition of sample buffer. Samples were then resolved by SDS-PAGE and visualized by autoradiography. Kinase assays for Far Westerns were performed as indicated above in the presence of nonradiolabeled ATP only. Far Westerns were done as previously described, using $1 \mu \mathrm{g} / \mathrm{mL}$ GST-tagged PBD (Neef et al. 2003). Cell extracts for all experiments were prepared in RIPA lysis buffer $(10 \mathrm{mM}$ Tris at $\mathrm{pH} 7.5,150 \mathrm{mM} \mathrm{NaCl}, 0.5 \%$ Triton $\mathrm{X}-100,1 \%$ sodium deoxycholate, $1 \mathrm{mM}$ sodium vanadate, $10 \mu \mathrm{g} / \mathrm{mL}$ of leupeptin per milliliter, $1 \mu \mathrm{g} / \mathrm{mL}$ Pepstatin $\mathrm{A}, 10 \mu \mathrm{g} / \mathrm{mL}$ aprotinin, and $1 \mathrm{mg} /$ $\mathrm{mL}$ Pefabloc). All Western blots and immunoprecipitations were performed with lysates equalized for protein content using the BCA assay (Pierce).

For pull-down experiments, $5 \mathrm{mg}$ of mitotic lysate were incubated for $1 \mathrm{~h}$ in the presence of $2 \mu \mathrm{g}$ of immobilized GST$\mathrm{PBD}^{\mathrm{WT}}$ or GST-PBD ${ }^{\mathrm{AA}}$ (Hanisch et al. 2006). After extensive washing, the beads were boiled in SDS-PAGE sample buffer and resolved by electrophoresis. BubR1 binding was then determined by Western blotting. Unless otherwise indicated, immunoprecipitations were performed with $1 \mu \mathrm{g}$ of antibody per milligram of cell extract.

Immunofluorescence microscopy and time-lapse videomicroscopy

Cells grown on coverslips were fixed and permeabilized simultaneously for $10 \mathrm{~min}$ at room temperature and processed for indirect immunofluorescence microscopy as described previously (Baumann et al. 2007). Samples were examined on a Deltavision microscope (Applied Precision), with optical sections acquired $0.15 \mu \mathrm{m}$ apart in the $Z$-axis. Deconvolved images from each focal plane were projected into a single picture using Softworx (Applied Precision). For time-lapse videomicroscopy, HeLa S3 cells stably expressing Histone H2B-GFP were used. During filming, cells were maintained in $\mathrm{CO}_{2}$-independent medium and at $37^{\circ} \mathrm{C}$ on a heated platform. Image acquisition was performed using a Zeiss Axiovert 2 microscope equipped with a Plan Neofluar 40x objective. Cells expressing both Histone H2B-GFP and mCherry-BubR1 were tracked for $16 \mathrm{~h}$. GFP images were acquired at 2-min intervals using Metamorph software (Visitron Systems), with mCherry monitored every tenth acquisition.

Detailed descriptions of other methods are available in the Supplemental Material.

\section{Acknowledgments}

We thank E. Chan, U. Grüneberg, A. Hanisch, T.U. Mayer, and R. Neef for reagents, and all members of the Nigg laboratory, especially U. Klein and C. Baumann, for insightful discussions. We also thank the MPIB core facility for peptide synthesis, S. Thiele for technical assistance, and U. Klein for critical reading of the manuscript. S.E. was supported by a Forschungsstipendium der Alexander von Humboldt-Stiftung, and currently holds a post-doctoral fellowship from the Canadian Institute of Health Research (CIHR). This work was supported by the Max Planck Society and the Fonds der chemischen Industrie. 


\section{References}

Abrieu, A., Kahana, J.A., Wood, K.W., and Cleveland, D.W. 2000. CENP-E as an essential component of the mitotic checkpoint in vitro. Cell 102: 817-826.

Ahonen, L.J., Kallio, M.J., Daum, J.R., Bolton, M., Manke, I.A., Yaffe, M.B., Stukenberg, P.T., and Gorbsky, G.J. 2005. Pololike kinase 1 creates the tension-sensing 3F3/2 phosphoepitope and modulates the association of spindle-checkpoint proteins at kinetochores. Curr. Biol. 15: 1078-1089.

Barr, F.A., Sillje, H.H., and Nigg, E.A. 2004. Polo-like kinases and the orchestration of cell division. Nat. Rev. Mol. Cell Biol. 5: 429-440.

Baumann, C., Korner, R., Hofmann, K., and Nigg, E.A. 2007. $\mathrm{PICH}$, a centromere-associated SNF2 family ATPase, is regulated by Plk1 and required for the spindle checkpoint. Cell 128: $101-114$.

Bharadwaj, R. and Yu, H. 2004. The spindle checkpoint, aneuploidy, and cancer. Oncogene 23: 2016-2027.

Chan, G.K., Schaar, B.T., and Yen, T.J. 1998. Characterization of the kinetochore binding domain of CENP-E reveals interactions with the kinetochore proteins CENP-F and hBUBR1. J. Cell Biol. 143: 49-63.

Chan, G.K., Jablonski, S.A., Sudakin, V., Hittle, J.C., and Yen, T.J. 1999. Human BUBR1 is a mitotic checkpoint kinase that monitors CENP-E functions at kinetochores and binds the cyclosome/APC. J. Cell Biol. 146: 941-954.

Chan, G.K., Liu, S.T., and Yen, T.J. 2005. Kinetochore structure and function. Trends Cell Biol. 15: 589-598.

Cheeseman, I.M., Chappie, J.S., Wilson-Kubalek, E.M., and Desai, A. 2006. The conserved KMN network constitutes the core microtubule-binding site of the kinetochore. Cell 127: 983-997.

Chen, R.H. 2002. BubR1 is essential for kinetochore localization of other spindle checkpoint proteins and its phosphorylation requires Mad1. I. Cell Biol. 158: 487-496.

Cimini, D., Wan, X., Hirel, C.B., and Salmon, E.D. 2006. Aurora kinase promotes turnover of kinetochore microtubules to reduce chromosome segregation errors. Curr. Biol. 16: 17111718.

Daum, J.R. and Gorbsky, G.J. 1998. Casein kinase II catalyzes a mitotic phosphorylation on threonine 1342 of human DNA topoisomerase II $\alpha$, which is recognized by the $3 \mathrm{~F} 3 / 2$ phosphoepitope antibody. J. Biol. Chem. 273: 30622-30629.

Daum, J.R., Tugendreich, S., Topper, L.M., Jorgensen, P.M., Hoog, C., Hieter, P., and Gorbsky, G.J. 2000. The 3F3/2 antiphosphoepitope antibody binds the mitotically phosphorylated anaphase-promoting complex/cyclosome. Curr. Biol. 10: R850-R852. doi: 10.1016/S0960-9822/00)00836-8.

DeLuca, J.G., Howell, B.J., Canman, J.C., Hickey, J.M., Fang, G., and Salmon, E.D. 2003. Nuf2 and $\mathrm{Hec} 1$ are required for retention of the checkpoint proteins Mad1 and Mad2 to kinetochores. Curr. Biol. 13: 2103-2109.

DeLuca, J.G., Gall, W.E., Ciferri, C., Cimini, D., Musacchio, A., and Salmon, E.D. 2006. Kinetochore microtubule dynamics and attachment stability are regulated by Hec1. Cell 127: 969-982.

Ditchfield, C., Johnson, V.L., Tighe, A., Ellston, R., Haworth, C., Johnson, T., Mortlock, A., Keen, N., and Taylor, S.S. 2003. Aurora B couples chromosome alignment with anaphase by targeting BubR1, Mad2, and Cenp-E to kinetochores. J. Cell Biol. 161: 267-280.

Dong, Y., Vanden Beldt, K.J., Meng, X., Khodjakov, A., and McEwen, B.F. 2007. The outer plate in vertebrate kinetochores is a flexible network with multiple microtubule interactions. Nat. Cell Biol. 9: 516-522.
Elia, A.E., Rellos, P., Haire, L.F., Chao, J.W., Ivins, F.J., Hoepker, K., Mohammad, D., Cantley, L.C., Smerdon, S.J., and Yaffe, M.B. 2003. The molecular basis for phosphodependent substrate targeting and regulation of Plks by the Polo-box domain. Cell 115: 83-95.

Gorbsky, G.J. 1995. Kinetochores, microtubules and the metaphase checkpoint. Trends Cell Biol. 5: 143-148.

Gorbsky, G.J. and Ricketts, W.A. 1993. Differential expression of a phosphoepitope at the kinetochores of moving chromosomes. J. Cell Biol. 122: 1311-1321.

Hanisch, A., Wehner, A., Nigg, E.A., and Sillje, H.H. 2006. Different Plk1 functions show distinct dependencies on PoloBox domain-mediated targeting. Mol. Biol. Cell 17: 448-459.

Hauf, S., Cole, R.W., LaTerra, S., Zimmer, C., Schnapp, G., Walter, R., Heckel, A., van Meel, J., Rieder, C.L., and Peters, J.M. 2003. The small molecule Hesperadin reveals a role for Aurora $\mathrm{B}$ in correcting kinetochore-microtubule attachment and in maintaining the spindle assembly checkpoint. J. Cell Biol. 161: 281-294.

Joseph, J., Liu, S.T., Jablonski, S.A., Yen, T.J., and Dasso, M. 2004. The RanGAP1-RanBP2 complex is essential for microtubule-kinetochore interactions in vivo. Curr. Biol. 14: 611-617.

Kang, Y.H., Park, J.E., Yu, L.R., Soung, N.K., Yun, S.M., Bang, J.K., Seong, Y.S., Yu, H., Garfield, S., Veenstra, T.D., et al. 2006. Self-regulated Plk1 recruitment to kinetochores by the Plk1-PBIP1 interaction is critical for proper chromosome segregation. Mol. Cell 24: 409-422.

King, E.M., Rachidi, N., Morrice, N., Hardwick, K.G., and Stark, M.J. 2007. Ipllp-dependent phosphorylation of Mad3p is required for the spindle checkpoint response to lack of tension at kinetochores. Genes \& Dev. 21: 1163-1168.

Kline-Smith, S.L., Sandall, S., and Desai, A. 2005. Kinetochorespindle microtubule interactions during mitosis. Curr. Opin. Cell Biol. 17: 35-46.

Kops, G.J., Weaver, B.A., and Cleveland, D.W. 2005. On the road to cancer: Aneuploidy and the mitotic checkpoint. Nat. Rev. Cancer 5: 773-785.

Lampson, M.A. and Kapoor, T.M. 2005. The human mitotic checkpoint protein BubR1 regulates chromosome-spindle attachments. Nat. Cell Biol. 7: 93-98.

Lenart, P., Petronczki, M., Steegmaier, M., Di Fiore, B., Lipp, J.J., Hoffmann, M., Rettig, W.J., Kraut, N., and Peters, J.M. 2007. The small-molecule inhibitor BI 2536 reveals novel insights into mitotic roles of polo-like kinase 1. Curr. Biol. 17: 304315.

Maiato, H., DeLuca, J., Salmon, E.D., and Earnshaw, W.C. 2004. The dynamic kinetochore-microtubule interface. J. Cell Sci. 117: 5461-5477.

Mann, M. 2006. Functional and quantitative proteomics using SILAC. Nat. Rev. Mol. Cell Biol. 7: 952-958.

Martin-Lluesma, S., Stucke, V.M., and Nigg, E.A. 2002. Role of $\mathrm{Hecl}$ in spindle checkpoint signaling and kinetochore recruitment of Mad1/Mad2. Science 297: 2267-2270.

Matsumura, S., Toyoshima, F., and Nishida, E. 2007. Polo-like kinase 1 facilitates chromosome alignment during prometaphase through BubR1. J. Biol. Chem. 282: 15217-15227.

McCleland, M.L., Kallio, M.J., Barrett-Wilt, G.A., Kestner, C.A., Shabanowitz, J., Hunt, D.F., Gorbsky, G.J., and Stukenberg, P.T. 2004. The vertebrate Ndc80 complex contains Spc24 and Spc25 homologs, which are required to establish and maintain kinetochore-microtubule attachment. Curr. Biol. 14: 131-137.

McEwen, B.F., Chan, G.K., Zubrowski, B., Savoian, M.S., Sauer, M.T., and Yen, T.J. 2001. CENP-E is essential for reliable bioriented spindle attachment, but chromosome alignment 
can be achieved via redundant mechanisms in mammalian cells. Mol. Biol. Cell 12: 2776-2789.

Meraldi, P., Draviam, V.M., and Sorger, P.K. 2004. Timing and checkpoints in the regulation of mitotic progression. Dev. Cell 7: 45-60.

Musacchio, A. and Hardwick, K.G. 2002. The spindle checkpoint: Structural insights into dynamic signalling. Nat. Rev. Mol. Cell Biol. 3: 731-741.

Neef, R., Preisinger, C., Sutcliffe, J., Kopajtich, R., Nigg, E.A., Mayer, T.U., and Barr, F.A. 2003. Phosphorylation of mitotic kinesin-like protein 2 by polo-like kinase 1 is required for cytokinesis. J. Cell Biol. 162: 863-875.

Neef, R., Gruneberg, U., Kopajtich, R., Li, X., Nigg, E.A., Sillje, H., and Barr, F.A. 2007. Choice of Plk1 docking partners during mitosis and cytokinesis is controlled by the activation state of Cdk1. Nat. Cell Biol. 9: 436-444.

Nigg, E.A. 2001. Mitotic kinases as regulators of cell division and its checkpoints. Nat. Rev. Mol. Cell Biol. 2: 21-32.

Nishino, M., Kurasawa, Y., Evans, R., Lin, S.H., Brinkley, B.R., and Yu-Lee, L.Y. 2006. NudC is required for Plk1 targeting to the kinetochore and chromosome congression. Curr. Biol. 16: 1414-1421.

Pinsky, B.A. and Biggins, S. 2005. The spindle checkpoint: Tension versus attachment. Trends Cell Biol. 15: 486-493.

Qi, W., Tang, Z., and Yu, H. 2006. Phosphorylation- and polobox-dependent binding of Plk1 to Bub1 is required for the kinetochore localization of Plk1. Mol. Biol. Cell 17: 37053716.

Rancati, G., Crispo, V., Lucchini, G., and Piatti, S. 2005. Mad3/ BubR1 phosphorylation during spindle checkpoint activation depends on both Polo and Aurora kinases in budding yeast. Cell Cycle 4: 972-980.

Rieder, C.L. 2005. Kinetochore fiber formation in animal somatic cells: Dueling mechanisms come to a draw. Chromosoma 114: 310-318.

Shaner, N.C., Campbell, R.E., Steinbach, P.A., Giepmans, B.N., Palmer, A.E., and Tsien, R.Y. 2004. Improved monomeric red, orange and yellow fluorescent proteins derived from Discosoma sp. red fluorescent protein. Nat. Biotechnol. 22: $1567-1572$.

Shannon, K.B., Canman, J.C., and Salmon, E.D. 2002. Mad2 and BubR1 function in a single checkpoint pathway that responds to a loss of tension. Mol. Biol. Cell 13: 3706-3719.

Sudakin, V., Chan, G.K., and Yen, T.J. 2001. Checkpoint inhibition of the APC/C in HeLa cells is mediated by a complex of BUBR1, BUB3, CDC20, and MAD2. J. Cell Biol. 154: 925936.

Sumara, I., Gimenez-Abian, J.F., Gerlich, D., Hirota, T., Kraft, C., de la Torre, C., Ellenberg, J., and Peters, J.M. 2004. Roles of polo-like kinase 1 in the assembly of functional mitotic spindles. Curr. Biol. 14: 1712-1722.

Tanudii, M., Shoemaker, J., L'Italien, L., Russell, L., Chin, G., and Schebye, X.M. 2004. Gene silencing of CENP-E by small interfering RNA in HeLa cells leads to missegregation of chromosomes after a mitotic delay. Mol. Biol. Cell 15: 37713781.

Taylor, S.S., Scott, M.I., and Holland, A.J. 2004. The spindle checkpoint: A quality control mechanism which ensures accurate chromosome segregation. Chromosome Res. 12: 599616.

Vagnarelli, P. and Earnshaw, W.C. 2004. Chromosomal passengers: The four-dimensional regulation of mitotic events. Chromosoma 113: 211-222.

van Vugt, M.A. and Medema, R.H. 2005. Getting in and out of mitosis with Polo-like kinase-1. Oncogene 24: 2844-2859.

van Vugt, M.A., van de Weerdt, B.C., Vader, G., Janssen, H.,
Calafat, J., Klompmaker, R., Wolthuis, R.M., and Medema, R.H. 2004. Polo-like kinase-1 is required for bipolar spindle formation but is dispensable for anaphase promoting complex/Cdc20 activation and initiation of cytokinesis. J. Biol. Chem. 279: 36841-36854.

Weaver, B.A., Bonday, Z.Q., Putkey, F.R., Kops, G.J., Silk, A.D., and Cleveland, D.W. 2003. Centromere-associated protein-E is essential for the mammalian mitotic checkpoint to prevent aneuploidy due to single chromosome loss. J. Cell Biol. 162: $551-563$.

Wong, O.K. and Fang, G. 2005. Plx1 is the 3F3/2 kinase responsible for targeting spindle checkpoint proteins to kinetochores. J. Cell Biol. 170: 709-719.

Wong, O.K. and Fang, G. 2006. Loading of the 3F3/2 antigen onto kinetochores is dependent on the ordered assembly of the spindle checkpoint proteins. Mol. Biol. Cell 17: 43904399.

Yao, X., Abrieu, A., Zheng, Y., Sullivan, K.F., and Cleveland, D.W. 2000. CENP-E forms a link between attachment of spindle microtubules to kinetochores and the mitotic checkpoint. Nat. Cell Biol. 2: 484-491. 


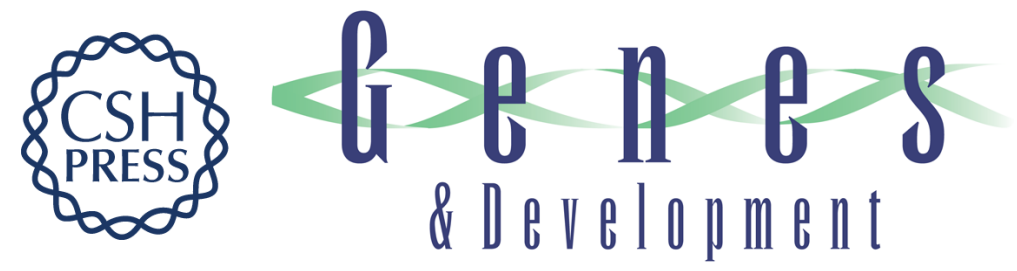

\section{Tension-sensitive Plk1 phosphorylation on BubR1 regulates the stability of kinetochore-microtubule interactions}

Sabine Elowe, Stefan Hümmer, Andreas Uldschmid, et al.

Genes Dev. 2007, 21:

Access the most recent version at doi:10.1101/gad.436007

Supplemental http://genesdev.cshlp.org/content/suppl/2007/08/16/21.17.2205.DC1
Material

References This article cites 58 articles, 22 of which can be accessed free at:

http://genesdev.cshlp.org/content/21/17/2205.full.html\#ref-list-1

License

Email Alerting Receive free email alerts when new articles cite this article - sign up in the box at the top

Service right corner of the article or click here.

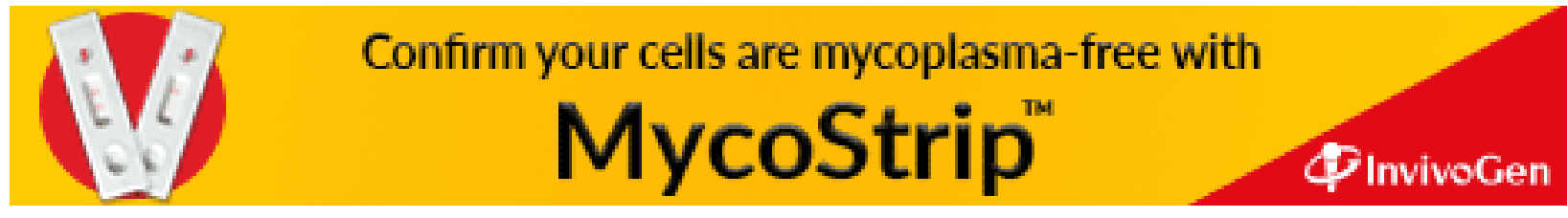

